\title{
Body Temperature as a Conditional Response Measure for Pavlovian Fear Conditioning
}

\author{
Bill P. Godsil, ${ }^{1,3}$ Jennifer J. Quinn, ${ }^{1}$ and Michael S. Fanselow ${ }^{1,2}$ \\ ${ }^{1}$ Department of Psychology, University of California, Los Angeles, California 90095-1563, USA; ${ }^{2}$ Brain Research Institute, University of \\ California, Los Angeles, California 90095-1761, USA; ${ }^{3}$ Interdepartmental Ph.D. Program in Neuroscience, University of California, \\ Los Angeles, California 90095-1761, USA
}

\begin{abstract}
On six days rats were exposed to each of two contexts. They received an electric shock in one context and nothing in the other. Rats were tested later in each environment without shock. The rats froze and defecated more often in the shock-paired environment; they also exhibited a significantly larger elevation in rectal temperature in that environment. The rats discriminated between each context, and we suggest that the elevation in temperature is the consequence of associative learning. Thus, body temperature can be used as a conditional response measure in Pavlovian fear conditioning experiments that use footshock as the unconditional stimulus.
\end{abstract}

Pavlovian fear conditioning is a model for the analysis of learning. In one version of this procedure a rat receives an aversive footshock while in a chamber. Later during a test, the rat is reexposed to the training environment, where it engages in behaviors that are characteristic of fear. The chamber, or context, serves as a conditional stimulus (CS). The context is originally a neutral stimulus, but elicits fear responses after it is paired with an unconditional stimulus (US), the footshock. Such responses to the CS are called conditional responses (CRs). These fear CRs occur specifically to the shock-paired context and not to other contexts that have not been paired with footshock. Fear CRs are used as measures of learning in Pavlovian experiments. To date, contextual fear has been assessed with several CRs such as defensive freezing, conditional analgesia, and conditional defecation. (Fanselow and Bolles 1979; Fanselow and Baackes 1982; Fanselow 1986).

Defensive freezing is a behavior characterized by the absence of all movement except that required for breathing. In the wild, rodents often freeze when they encounter a predator. This behavior is an effective defensive strategy because many predators have difficulty detecting an immobile target, and movement can act as a releasing stimulus for predatory attacks (Fanselow and Lester 1988). In the laboratory, freezing is prevalent when rodents are presented with a context that has been paired with footshock (Fanselow and Bolles 1979). Freezing provides a behavioral index of learning that is amenable to parametric analysis, and it can be assessed in freely moving animals without the administration of extraneous test stimuli that may affect

${ }^{3}$ Corresponding author.

EMAIL godsil@lifesci.ucla.edu; FAX (310) 206-5895.

Article and publication are at www.learnmem.org/cgi/doi/ $10.1101 / \mathrm{lm} .32800$. behavior directly (e.g., startle stimuli, painful stimuli). For these reasons, freezing has become a popular measure of fear.

Certainly, however, fear is characterized by behaviors other than freezing. For example, the rat becomes less sensitive to pain when it faces fear-provoking situations (e.g., Fanselow and Baackes 1982). Similarly, a rat will show an enhanced startle reflex in the presence of fear CSs (Davis 1986). Although useful, these measures of fear share a disadvantage: both require the administration of a strong stimulus to produce a baseline of behavior for fear to be detected. Conditional analgesia requires a painful stimulus such as a formalin injection to generate baseline recuperative behavior, and potentiated startle uses a loud noise probe to generate the baseline startle response. Several studies have shown that this startle stimulus itself can act as a US capable of conditioning fear in much the same way that a shock can (e.g., Borszcz et al. 1989). Consequently, every potentiated startle test session is also a new acquisition session.

Conditional fear also has been assessed by monitoring defecation. Rats defecate more often when exposed to a shock-paired context (Fanselow 1986). Similarly, the freely moving rat's heart rate and blood pressure increase when reexposed to an aversive tone CS (Iwata and LeDoux 1988), and rats vocalize to an aversive light CS (Nitschke 1979). Thus, conditional fear can be measured with a variety of CRs.

Changes in body temperature have been reported previously to be CRs in other Pavlovian experiments. Such a CR was detected when morphine was used as a US for conditional tolerance (Eikelboom and Stewart 1979) and when lipopolysaccharide (LPS) was used as the US in a taste aversion experiment (Bull et al. 1991). Previous investigations that have used temperature as a measure of fear have gen-

LEARNING \& MEMORY 7:353-356 @ 2000 by Cold Spring Harbor Laboratory Press ISSN1072-0502/00 \$5.00

$$
\begin{array}{lllllllllllllll}
\text { L } & E & A & R & N & I & N & G & \mathcal{Z} & M & E & M & O & R & Y \\
\text { www.learnmem.org } & & &
\end{array}
$$


erated ambiguous results (Antoniadis and McDonald 1999, 2000). We hypothesized that this response could also be detected in experiments that use footshock as the US. The purpose of this investigation was to determine if another behavioral index, core body temperature, could serve as a useful index of contextual fear learning.

\section{MATERIALS AND METHODS}

Eight adult male Long-Evans rats (400-500 g), bred and raised at UCLA, were housed in individual cages on Day 1 of the experiment. Animals had free access to food and water and were kept on a 14:10 day/night cycle. All experimental procedures began three hr after light onset and ended two $\mathrm{hr}$ later. The room temperature was $22^{\circ} \mathrm{C} \pm 1.5^{\circ} \mathrm{C}$.

On Days 2-6 the experimenter handled the animals in the vivarium as they were habituated to the insertion of a rectal thermistor probe (Cole-Parmer Instrument Company, Chicago, IL). The probe was $3.3 \mathrm{~mm}$ in diameter, and was lubricated with petroleum jelly. Temperature measurement was conducted on a table. Each rat was held at the base of its tail as the probe was inserted $\sim 2.5 \mathrm{~cm}$ into the rectum. The probe was removed after the animal's temperature stabilized $30-60 \mathrm{sec}$ later. The thermistor was accurate to $0.1^{\circ} \mathrm{C}$.

Two highly discriminable contexts were used in this experiment. The shock-paired context chamber was an aluminum and Plexiglas chamber (aluminum sides, translucent top and back, transparent front; $28 \mathrm{~cm}$ wide, $21 \mathrm{~cm}$ high and $22 \mathrm{~cm}$ deep; Lafayette Instruments, Lafayette, IN). The floor of the chamber had 18 stainless steel rods ( $4 \mathrm{~mm}$ diameter, $1.5 \mathrm{~cm}$ apart) connected to a shock scrambler (machine-shop, UCLA) and generator (Lafayette Instruments) which, along with ventilation fans, provided background noise $(70 \mathrm{~dB}$, A scale). The chamber was scented with $5 \%$ ammonium hydroxide (in collection pan below rods). This chamber was in a well-lit room separate from the observers, who viewed animals on a monitor. The no-shock context chamber was in a separate laboratory room. This chamber (same dimensions as described earlier) had a white rear wall and white Plexiglas side walls $(24 \times 21 \mathrm{~cm})$ placed at $60^{\circ}$ angles to the floor, forming a triangular enclosure that made the internal volume of this context less than that of the shock-paired context. The floor consisted of 17 staggered rods (two rows, $1 \mathrm{~cm}$ vertically apart; within a row, each rod was $2.6 \mathrm{~cm}$ apart). A white noise generator supplied background noise $(70 \mathrm{~dB})$, and the chamber was scented with $1 \%$ acetic acid. This room was kept dark, except for a single $30 \mathrm{~W}$ red light bulb. Rats exhibit little generalization between these contexts (e.g., Kim and Fanselow 1992).

Animals received six consecutive days of discrimination training (Days 7-12) followed by a single testing day (Day 13). Each animal was exposed to both contexts for the same amount of time each day of the fear conditioning procedures. On the first day, training began in the shock-paired context. Thereafter, the first context to be used alternated on each subsequent day. During each day of conditioning the animals were transported in their home cages to a holding room adjacent to the experimental rooms. In a random order, a single animal was carried to either the shockpaired or no-shock context. The animal's temperature was measured before placement and after removal from the chamber. Animals trained in the shock-paired context received a single footshock ( $2 \mathrm{sec}, 0.71 \mathrm{~mA}$, measured across the grid with a $50-\mathrm{K} \Omega$ resistor) $192 \mathrm{sec}$ after placement in the chamber. They were removed from the chamber $30 \mathrm{sec}$ after termination of footshock. Animals trained in the no-shock context were placed in the cham- ber for $224 \mathrm{sec}$ before they were removed. Rats were returned to their home cage until they were trained in the other environment approximately one $\mathrm{hr}$ later. On the testing day, rats were treated as described earlier except that animals did not receive a shock in the shock-paired context: each rat was exposed to the shock-paired context and no-shock context for $224 \mathrm{sec}$. Half of the animals were tested in the shock-paired context first; the remaining animals were tested in the no-shock context first.

Three measures were taken. Freezing was defined as the absence of any visible movement except that required for breathing (fluctuation of the chest cavity). Freezing was scored for the first $192 \mathrm{sec}$ of each exposure to either chamber (across 6 days of training and 1 day of testing). Freezing was scored according to an instantaneous time-sampling procedure in which each animal was observed every eight sec for a 192 -sec interval. These observations yielded an estimate of the percentage time spent freezing. Fecal boli counts were taken after each test. The experimenter would count pellets left by the animal in the conditioning chamber. Body temperature was computed as the difference in temperature measured before placement and after removal from the conditioning chamber. The probe was left in until the animal's temperature stabilized (30-60 sec, typically). The table used during habituation was wheeled into each conditioning room for use in the temperature-measurement procedure.

A repeated-measure ANOVA was used to analyze the freezing acquisition data. Paired $t$-tests were used to analyze the freezing, body temperature, and defecation test data. For these comparisons, $\alpha$ was set at 0.05 .

One rat exhibited high levels of freezing, defecation, and increases in body temperature in both contexts on the training and test days. These data (not shown) suggest that the animal did not learn the context discrimination, and they were excluded from the analyses. Thus, the analyses are taken from the data of the remaining seven rats.

Figure 1 depicts freezing data collected during training. Freezing was scored for $192 \mathrm{sec}$ in both the shock-paired and no-shock contexts. This time period is the duration of the preshock period in the shock context. Freezing was negligible on the first day of training. Acquisition showed the classic discrimination learning pattern: there was a negatively accelerated acquisition of the CR over days in the shock-paired context. In the no-shock context there was some freezing that rapidly decreased over days. This pattern was confirmed with a two-factor repeated-measure analysis of variance (ANOVA). This analysis revealed a significant day $x$ context interaction during the six days of training $\left(F_{5,84}=17.06, P<0.001\right)$. Post-hoc comparisons with Scheffe's test indicated that freezing was reliably different in the contexts on all but Day 1 of training. Temperature and defecation had to be assessed after the rats were removed from the chamber. During acquisition these measures were confounded with the unconditional effects of the shock. Thus, these measures were not reported for the acquisition sessions.

Figure 2A depicts freezing data collected during the shockfree test in each context. On average, the rats froze $82 \%$ of the time in the shock-paired context and 5\% of the time in the no-shock context. A paired $t$-test revealed a significant difference across the two contexts $\left(t_{6}=35.165, P<0.001\right)$. Thus, based on this freezing measure, the contexts were highly discriminable on the test day. Consistent with prior studies, the shock-associated context showed differential control over freezing.

Figure 2B depicts defecation data collected during the test day in each context. On average, the rats excreted 4.1 pellets in the shock-paired context and 0.3 pellets in the no-shock context. A

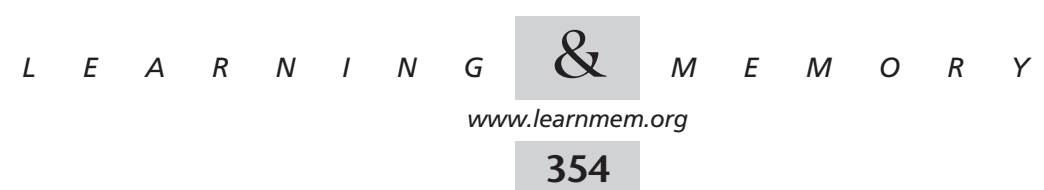




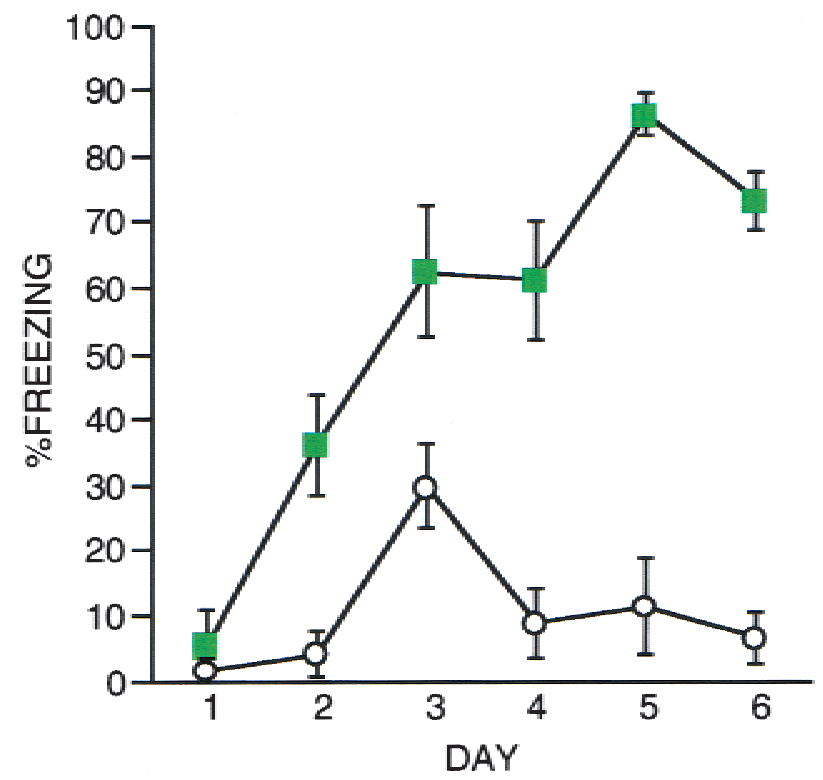

Figure 1 Pavlovian fear conditioning acquisition across the six training days. Freezing was scored for the $192 \mathrm{sec}$ before shock in each context on each day. Rats exhibited a gradual discrimination of the contexts across the training days. Group means and standard errors are shown. Shock-paired context (solid square); No-shock context (open circle).

paired $t$-test revealed a significant difference in pellet output across contexts $\left(t_{6}=4.361, P<0.005\right)$. Thus, the contexts were highly discriminable with this measure on the test day. The defecation measure was sensitive to the different levels of fear in the two contexts.

Figure 2C depicts the change in temperature detected in both contexts on the test day. Data are represented as the average change in temperature in each context. On average, rats exhibited a $0.96^{\circ} \mathrm{C}$ increase in body temperature in the shock-paired context and a $0.51{ }^{\circ} \mathrm{C}$ increase in body temperature in the no-shock context. A paired $t$-test was used to compare these changes in temperature, and it revealed a significant difference between contexts on the test day $\left(t_{6}=3.137, P<0.05\right)$. Thus, with this measure, the contexts were discriminable.

In this study, we investigated whether changes in body temperature can be used as a conditional response measure in a Pavlovian fear conditioning experiment. Animals placed in a context that had previously been paired with shock exhibited significant increases in body temperature when compared with reexposure to a no-shock context. This body temperature measure was consistent with other CR measurements because the animals also displayed significantly higher levels of freezing and defecation in the shockpaired context. Rats show no differences between these contexts during acquisition or testing if they are not shocked (data not shown). Thus, the present findings suggest that the increase in body temperature was the result of associative learning: the rats learned to associate the shock-paired chamber with the occurrence of shock, and this association triggered an elevation in body temperature in the shock-paired context. Thus, an increase in body temperature is a component of the constellation of CRs that rats exhibit to aversive conditional stimuli.

Changes in body temperature previously have been shown to be CRs in other Pavlovian paradigms (e.g., Eikelboom and Stewart 1979; Bull et al. 1991). In these examples, morphine or LPS acted as the US. Our present findings demonstrate that this CR can be detected in experiments that use footshock as the US.

In a recent study, Antoniadis and McDonald (1999) used chronic intraperitoneal temperature-monitoring devices during a fear conditioning experiment. These investigators reported a trend of elevated body temperature in the shock-paired context during

A

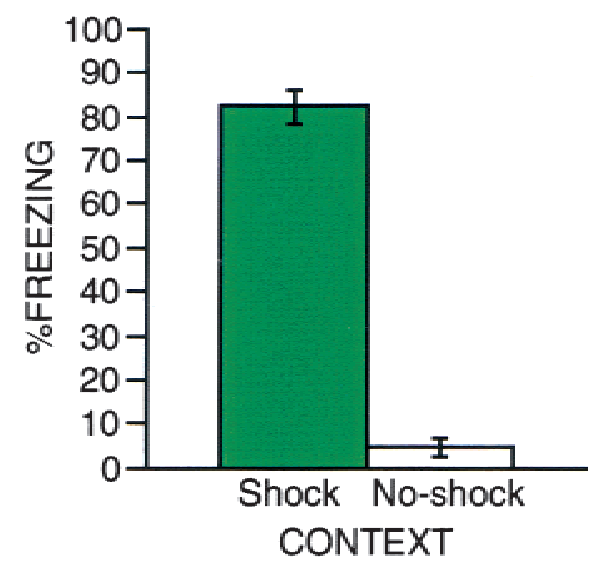

B

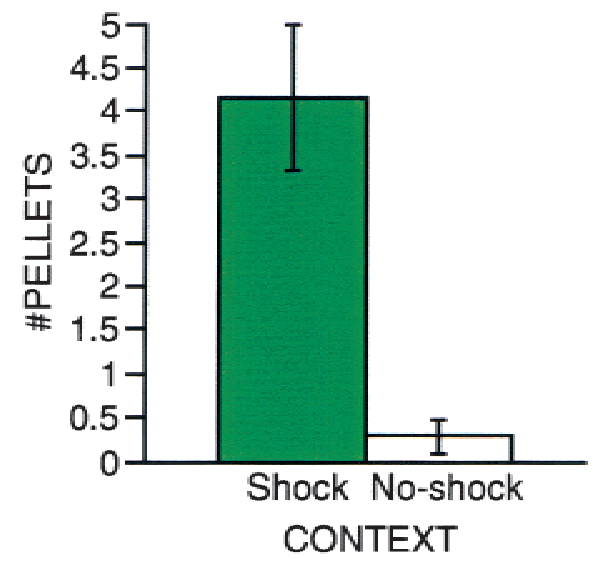

C

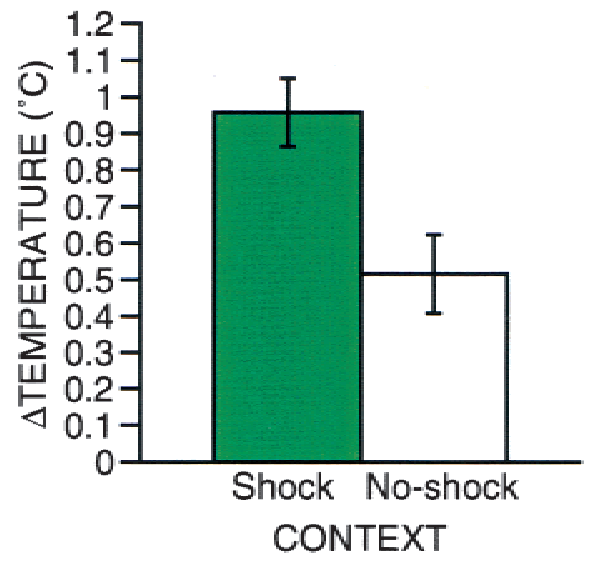

Figure 2 Pavlovian fear conditioned freezing, defecation, and temperature data from the test sessions. Rats exhibited discriminative freezing and defecation. They also exhibited an elevated change in body temperature in the shock-paired context. Group means and standard errors are shown. (A) Conditional freezing data. (B) Defecation data. (C) Body temperature data.

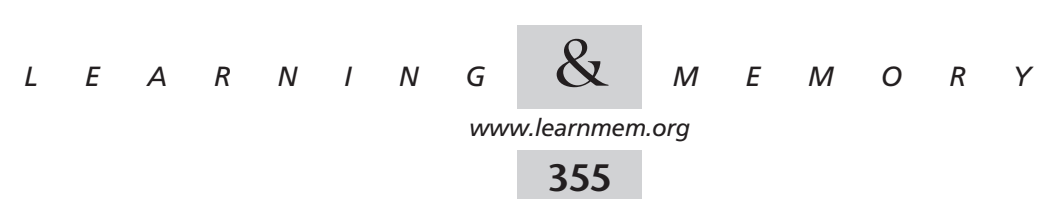


testing (three tests in each context; 10, 10, and 20 min in duration, respectively), and they speculated that this trend might become statistically reliable if their test duration was extended. In a more recent report, this hypothesis was tested, but rats exhibited a significant elevation in body temperature in the no-shock context (Antoniadis and McDonald 2000). Here we report a statistically reliable elevation in body temperature when rats are reexposed to a shock-paired context. Thus, our results are consistent with Antoniadis and McDonald (1999) and inconsistent with Antoniadis and McDonald (2000).

Antoniadis and McDonald $(1999,2000)$ designed their discriminative conditioning procedure such that it did not maximize differences between the shock-paired and no-shock contexts. Instead, their apparatus had an intervening corridor connecting the two chambers, and animals were permitted to explore the entire apparatus before conditioning. With this procedure, subjects exhibited high levels of freezing in both environments during the tests $(\sim 76 \%$ vs. $47 \%$ freezing in the paired and unpaired, respectively; Antoniadis and McDonald 1999). Thus, although these investigators detected a statistically significant discrimination, there was substantial generalization across contexts. In contrast, our conditioning procedure promoted maximum discrimination between environments because the two contexts differed across several dimensions. Consequently, the difference in the magnitude of freezing in the two contexts was large ( $82 \%$ vs. $5 \%$ freezing in the paired and unpaired, respectively). Thus, our procedure may have been more sensitive in detecting changes in temperature. Also, our training protocol was spread over six days, whereas Antonidias and McDonald used a two-day training procedure; a body temperature CR may require more trials over more days to become detectable. Another difference is the time at which temperature was assessed. Our measurements were taken approximately four min after placement in the chamber, whereas Antoniadis and McDonald's (2000) data were collected during the first $10 \mathrm{~min}$ of a third 20-min test session. Perhaps fearful rats are initially hyperthermic, but later show a rebound hypothermia.

In conclusion, reexposure to a shock-paired context prompts a reliable elevation in body temperature in the rat. This CR, core body temperature, may be a useful measure of fear. It should be noted that rectal temperature measurement has drawbacks (the probe is a stress-inducing stimulus). This CR could become especially useful with the development of less-invasive means of temperature measurement (infrared camera system, telemetry, etc.).

\section{ACKNOWLEDGMENTS}

This work was supported by NSF IBN9723295 and 444040FA34323 to M.S.F. and NRSA MH12402 to B.P.G. We thank A. Kirszenbaum,
L. Kirszenbaum, and S. Mellbom for their assistance and M.J. Sanders for his comments on the manuscript.

The publication costs of this article were defrayed in part by payment of page charges. This article must therefore be hereby marked "advertisement" in accordance with 18 USC section 1734 solely to indicate this fact.

\section{REFERENCES}

Antoniadis, E.A. and McDonald, R.J. 1999. Discriminative fear conditioning to context expressed by multiple measures of fear in the rat.Behav. Brain Res. 101: 1-13.

- 2000. Amygdala, hippocampus, and discriminative fear conditioning of context. Behav. Brain Res. 108: 1-19.

Borszcz, G.S., Cranney, J., and Leaton, R.N. 1989. Influence of long-term sensitization on long-term habituation of the acoustic startle response in rats: Central gray lesions, preexposure, and extinction. J. Exp. Psychol. Anim. Behav. Process 15: 54-64.

Bull, D.F., Brown, R., King, M.G., and Husband, A.J. 1991. Modulation of body temperature through taste aversion conditioning. Physiol. Behav. 49: 1229-1233.

Davis, M. 1986. Pharmacological and anatomical analysis of fear conditioning using the fear-potentiated startle paradigm. Behav. Neurosci. 100: 814-824.

Eikelboom, R. and Stewart, J. 1979. Conditioned temperature effects using morphine as the unconditioned stimulus. Psychopharmacology 61: 31-38.

Fanselow, M.S. 1986. Associative vs. topographical accounts of the immediate-shock freezing deficit in rats: Implications for the response selection rules governing species-specific defensive reactions. Learning and Motivation 17: 16-39.

Fanselow, M.S. and Baackes, M.P. 1982. Conditioned fear-induced opiate analgesia on the formalin test: Evidence for two aversive motivational systems. Learning and Motivation 13:200-221.

Fanselow, M.S. and Bolles, R.C. 1979. Naloxone and shock-elicited freezing in the rat. J. Comp. Physiol. Psychol. 93:736-744.

Fanselow, M.S. and Lester, L.S. 1988 A functional behavioristic approach to aversively motivated behavior: Predatory imminence as a determinant of the topography of defensive behavior. In Evolution and Learning (ed. R.C. Bolles, and M.D. Beecher), pp.185-212. Lawrence Erlbaum Associates, Inc., Hillsdale, NJ.

Iwata, J. and LeDoux, J.E. 1988. Dissociation of associative and nonassociative concomitants of classical fear conditioning in the freely behaving rat. Behav. Neurosci. 102: 66-76.

Kim J.J. and Fanselow, M.S. 1992. Modality-specific retrograde amnesia of fear. Science 256: 675-677.

Nitschke, W. 1979. Classical conditioning of vocalizations in the rat: Acoustic characteristics of the CR and UCR. Anim. Learn. E Behav. 7: 457-460.

Received April 25, 2000; accepted in revised form July 26, 2000.

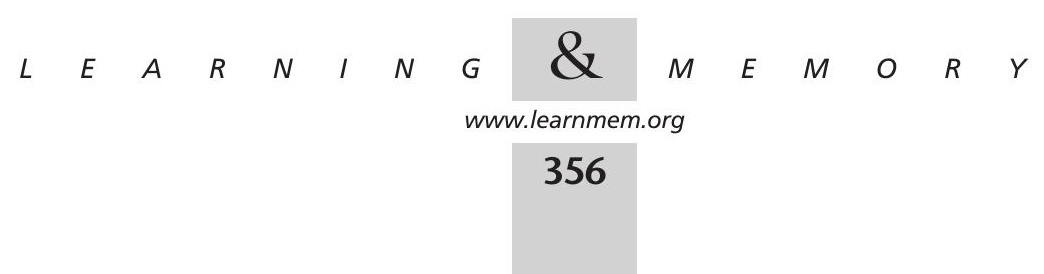




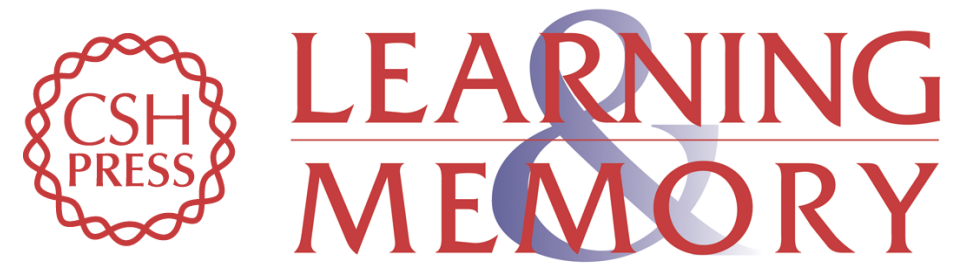

\section{Body Temperature as a Conditional Response Measure for Pavlovian Fear Conditioning}

Bill P. Godsil, Jennifer J. Quinn and Michael S. Fanselow

Learn. Mem. 2000, 7:

Access the most recent version at doi:10.1101//m.32800

References This article cites 36 articles, 1 of which can be accessed free at:

http://learnmem.cshlp.org/content/7/5/353.full.html\#ref-list-1

License

Email Alerting Receive free email alerts when new articles cite this article - sign up in the box at the Service top right corner of the article or click here. 